\title{
Anisotropic scaling of remotely sensed drainage basins: the differential anisotropy scaling technique
}

\author{
A. Beaulieu ${ }^{1, *}$, H. Gaonac'h ${ }^{1}$, and S. Lovejoy ${ }^{2}$ \\ ${ }^{1}$ Université du Québec à Montréal - Centre GEOTOP-UQÀM-McGill, C.P.8888 succursale Centre-Ville, Montréal, Québec, \\ H3C 3P8, Canada \\ ${ }^{2}$ McGill University, Department of Physics, 3600 University, Montreal, Québec, H3A 2T8, Canada \\ *now at: Centre for Topographic Information in Sherbrooke, Natural Resources Canada, 2144 King Street West, suite 010, \\ Sherbrooke, Qc, J1J 2E8, Canada
}

Received: 7 February 2007 - Revised: 16 May 2007 - Accepted: 16 May 2007 - Published: 6 July 2007

\begin{abstract}
We investigate the statistical properties of dendritic drainage areas from diverse geological environments (Deception Canyon, Utah and the Loess Plateau, China) using narrow band visible ASTER satellite images. We show that from $240 \mathrm{~m}$ to $7680 \mathrm{~m}$, the isotropic (angle integrated) energy spectra $E(k)$ of all the fields closely follow a power law form: $E(k) \propto k^{-\beta}$ where $k$ is a wave number and $\beta$ a scale invariant exponent. In spite of this good isotropic scaling, images with very similar $\beta$ 's and similar isotropic multifractal exponents have distinct textures; we suggest that the differences are primarily due to anisotropy, which is nevertheless scaling. We develop the new "Differential Anisotropy Scaling" technique to characterize this scale-by-scale (differential) anisotropy and we test it on simulated anisotropic scaling fields. The method gives useful characterizations of the scale by scale anisotropy irrespective of whether or not the analyzed field is scaling. When the anisotropy is not too strong, the parameters can be interpreted as scale invariant anisotropy exponents. Viewed as a method of estimating these exponents, it has the advantage of relying on two linear regressions rather than on complex higher dimensional nonlinear ones. When applied to dendritic drainage basins we find that they have distinct anisotropies characterized by differential anisotropy stretching and rotation parameters as well as by a distinct absolute anisotropy at the reference scale of $960 \mathrm{~m}$. Our new method allows us to statistically distinguish, not only between two geologically different drainage basins (the China Loess Plateau and Utah Deception Canyon), but also between different regions of the same China drainage system.
\end{abstract}

Correspondence to: A. Beaulieu

(abeaulie@nrcan.gc.ca)

\section{Introduction}

The Earth's surface is the product of tectonic, volcanic and erosion/deposition processes. The resulting complex landforms and the evolution of the surface are thus the consequence of nonlinear processes, some of which are catastrophic. The same processes apparently repeat from one scale to another, producing natural structures such as mountains, streams, lava flows and islands which have structures over wide ranges of scale. Quantitative measurements of these geomorphological features (e.g., lengths, perimeters, areas, roughness) or their associated topographic fields are found to be power law functions of their size or scale/resolution of acquisition (e.g., Lavallée et al., 1993; Gaonac'h et al., 1992; Gagnon et al., 2006): from meters to tens of kilometers they are often scale invariant. It is then important to link the observed scale invariant properties with the underlying physical and chemical processes.

Erosion of the Earth's surface on global scales is primarily caused by surface run-off. Fluvial landforms are peculiar structures with diverse drainage systems involving networks of small brooks to large rivers that evolve over time. A number of scaling investigations of river networks have attempted to relate unique exponents to the network topology such as stream orders and stream lengths (Horton, 1945; La Barbera and Rosso, 1989; Chase, 1992; Pelletier, 1999; Rodriguez-Iturbe and Rinaldo, 2001). However, investigations of geophysical fields rather than geometric drainage patterns are necessary for characterizing and understanding the geo-processes involved in shaping the Earth. Whereas scaling geometric sets may be described with unique exponents, scaling geophysical fields require nonlinear exponent functions. These multifractal exponent functions are generally necessary to specify the statistical moments of all orders (e.g., Lovejoy and Schertzer, 1990; Lavallée et al., 1993;

Published by Copernicus Publications on behalf of the European Geosciences Union and the American Geophysical Union. 
Weissel and Pratson, 1994; Pecknold et al., 1997; Veneziano and Iacobellis, 1999; Gagnon et al., 2006) hence to completely characterize the statistical scale dependence of the multifractal fields. The characterization of geofields by their scaling exponents is predicated on the fact that geotextures, georoughness and other basic aspects of geomorphology are fundamentally multiscale notions requiring wide ranges of scale for their expression. The acquisition of remotely sensed multiple spectral images is thus a unique source of data for determining the scale invariant characteristics of the radiance fields (e.g. visible, short-wave infrared, infrared, microwave) related to the chemical composition of soil and bedrock, their humidity content, their surface temperature (e.g., Laferrière and Gaonac'h, 1999; Maître and Pinciroli, 1999; Lovejoy et al., 2001a, b; Harvey et al., 2002; Beaulieu and Gaonac'h, 2002; Gaonac'h et al., 2003; Cheng, 2004).

The simplest scale invariance hypothesis is that the statistics are independent of direction; the system is isotropic, "self-similar". Until now, the scale invariant properties of surfaces have been routinely characterized using such isotropic statistics, which disregard (or "wash out") the effect of anisotropy or preferentially oriented structures by integrating or averaging statistics over all directions. For example, Gagnon et al. (2006) found over a range of $40 \mathrm{~m}$ to $20000 \mathrm{~km}$, for statistical moments of order lower than 2 , the residuals were on average within $\pm 45 \%$ of the theoretical isotropic values. They concluded that the observed tremendous diversity of geomorphologies is a consequence of anisotropy.

Geoanisotropy is expected to be a function of both size and location. Rather than imposing an a priori isotropic approach - e.g. by defining the size using the usual isotropic (Euclidean) distance function - it is more appropriate to allow the system itself to define the physically appropriate anisotropic notion of scale. We therefore seek to relate morphology and texture with anisotropic scaling. Up until now, only a few studies have investigated scaling anisotropy and most of these have only considered anisotropy in orthogonal directions (e.g. Lavallée et al., 1993; Weissel et al., 1995; Foufoula-Georgiou and Sapozhnikov, 1998). However, this is an insufficient generalization of the anisotropic scaling for most applications. Schertzer and Lovejoy (1985) proposed a general formalism for scale invariance where the anisotropy changes in direction and intensity via a scale-changing operation; see Pflug et al. (1993), Pecknold et al. (1997) and Lewis et al. (1999) for attempts at more general scaling characterizations of empirical fields. In the case where the anisotropy is only a function of size (but not location) the appropriate notion of scale for these structures is determined by a power law where the exponent is a matrix instead of a scalar. This matrix $\mathbf{G}$, generator of the anisotropy, determines the notion of scale and hence the differential scaling according to the directions (for example see Lovejoy et al., 1992, 2001a, b, 2005; Pflug et al., 1993; Pecknold et al., 2001; Lilley et al., 2004; Cheng, 2004). But anisotropy is important for im- age processing and pattern recognition too. For example the investigation of the anisotropy of fluvial regions by remote sensing is essential to broadly characterize the effect of the nonlinear processes which create drainage patterns in various environments.

In the present study, remotely sensed images from the TERRA ASTER sensor give us an opportunity to analyze the isotropic and anisotropic scaling statistical properties of drainage basins in different lithological backgrounds: the China Loess Plateau and the Utah Desolation Canyon (presented in Sect. 2); this raises a few questions. Does the anisotropy of drainage basins break the scaling of the satellite images? Do stream systems evolving in different geological settings show scaling anisotropies? In Sect. 3, we compare the isotropic energy spectra demonstrating scale invariant characteristics of fluvial textures. In Sect. 4, we consider the anisotropic scaling of the images through a new "Differential Anisotropy Scaling" technique that characterizes the differential anisotropy versus the scale of the observed fluvial structures testing it first on simulated multifractal anisotropic scaling fields and then on real images. We then discuss the resulting statistical similarities and differences and relate them with distinct drainage areas formation caused by different underlying Earth processes. In Sect. 5 we conclude.

\section{The data}

Remotely sensed images from the TERRA ASTER sensor (level 1A reconstructed unprocessed instrument data) in band $2(0.63-0.69 \mu \mathrm{m})$ were selected over two drainage areas. This particular spectral band was chosen due to its low atmospheric diffusion and hence high contrast. The selected images were relatively free of urban areas. They have vegetation-free homogeneous lithologies and relatively dry climates. To reduce high frequency banding (due to sensor calibration imperfections), the images (45 in total) were averaged to $512 \times 512$ pixels resulting in a spatial resolution of $30 \times 30 \mathrm{~m}$.

Images were chosen over the China Loess Plateau area on the Yellow River (Huang He, CL and CY, Fig. 1a) drainage basin west of Luliang Mountains and in the west part of the Taihang Mountains (CM, Fig. 1a). The CL group contains 17 images collected on 1 October 2000, the CY group contains 5 images from the same ASTER scenes, the CM group contains 16 images collected on 10 May 2001. The formation of the Loess Plateau in China dates back to 7-8 Ma, but since $2.6 \mathrm{Ma}$ the loess accumulation was very rapid (DerbyShire, 2001). The average grain size and thickness diminish from NW to SE, reflecting in a general way the dynamics of the transport of the loess by the dominant winds. Deposition of particles from the atmosphere produces very loose particle packing (Derbyshire, 2001). The stability of the loess deposit is sustained over a large area mainly because of climatic 
conditions; the dry climate maintains under-saturated conditions. The effect of rain on loess is drastic; water saturation causes it to disaggregate nearly instantaneously, creating landslides and mudflows. The section selected over the Yellow River (CL and CY) is mainly composed of loess deposits down to a depth of several meters (Porter, 2001). This area of the loess plateau displays a dendritic type river network, demonstrating the great homogeneity of the loess deposits being expressed as a "superfine texture". Such a drainage network could be caused by a poorly permeable surface deposit such as loess where conditions of intense but infrequent rainfalls prevail. Images chosen in this superfine texture area are separated in two groups $\mathrm{CL}$ and $\mathrm{CY}$. The $\mathrm{CL}$ images are predominantly composed of small to medium sized tributaries of the Yellow River (Fig. 2a) while the CY images include the more developed large Yellow River valley (Fig. 2b). The CM group with thinner loess deposits containing more clay was chosen from the Eastern part of the Loess Plateau near the Taihang Mountains (Fig. 2c). This dendritic area shows a "fine texture" (not superfine) with small less dense tributaries compared to the area west of the Luliang Mountains (possibly due to the superimposed effect of the thinner loess deposit in this area and the more permeable underlying hard rock basement). This Taihang Mountain region is mainly composed of granite and gneiss (Zunyi et al., 1986). All of these regions are in semi-arid conditions, and receive $300-600 \mathrm{~mm} / \mathrm{yr}$ of precipitation with mean annual temperatures of $2-6^{\circ} \mathrm{C}$ (Wang and Takahashi, 1998). The effect of sudden intense rainfalls may be very different on the CL and CY loess regions compared to the CM loess-granite-gneiss region.

Another region chosen was Desolation Canyon, Utah, (Fig. 1b); a selected image is shown in Fig. 2d (UT). The UT group contains 7 images acquired on 19 October. The Green River cuts deeply into this area of the Tavaputs Plateau (mainly the Roan Cliffs and the Desolation Canyon) where $5000 \mathrm{~m}$ of sediments were deposited in the ancient "Uinta Basin" paleogene deposits which are formed by a Laramide downwarp formed by structural disruption of the foreland region during the late Cretaceous and Paleogene epochs (Dickinson et al., 1986; Franczyk et al., 1991). The sediments are mainly composed of interbedded formations of shale, siltstone and sandstone. The whole region including the Tavaputs Plateau has witnessed regular tectonic uplift and erosion/deposition in the past $20 \mathrm{Ma}$ influencing the pathway of river drainage systems. The Green River developed in this complex geological environment also tends to be dendritic, but with an even lower drainage density, structurally controlled by diverse permeable rock formations and tectonic evolution of the Tavaputs Plateau called a "medium texture". Although the climate in this area is similar to the Chinese regions - a semi-arid environment receiving $300-400 \mathrm{~mm}$ of mean annual precipitation and mean annual temperatures of $1-7^{\circ} \mathrm{C}-$ the geological setting is very different.
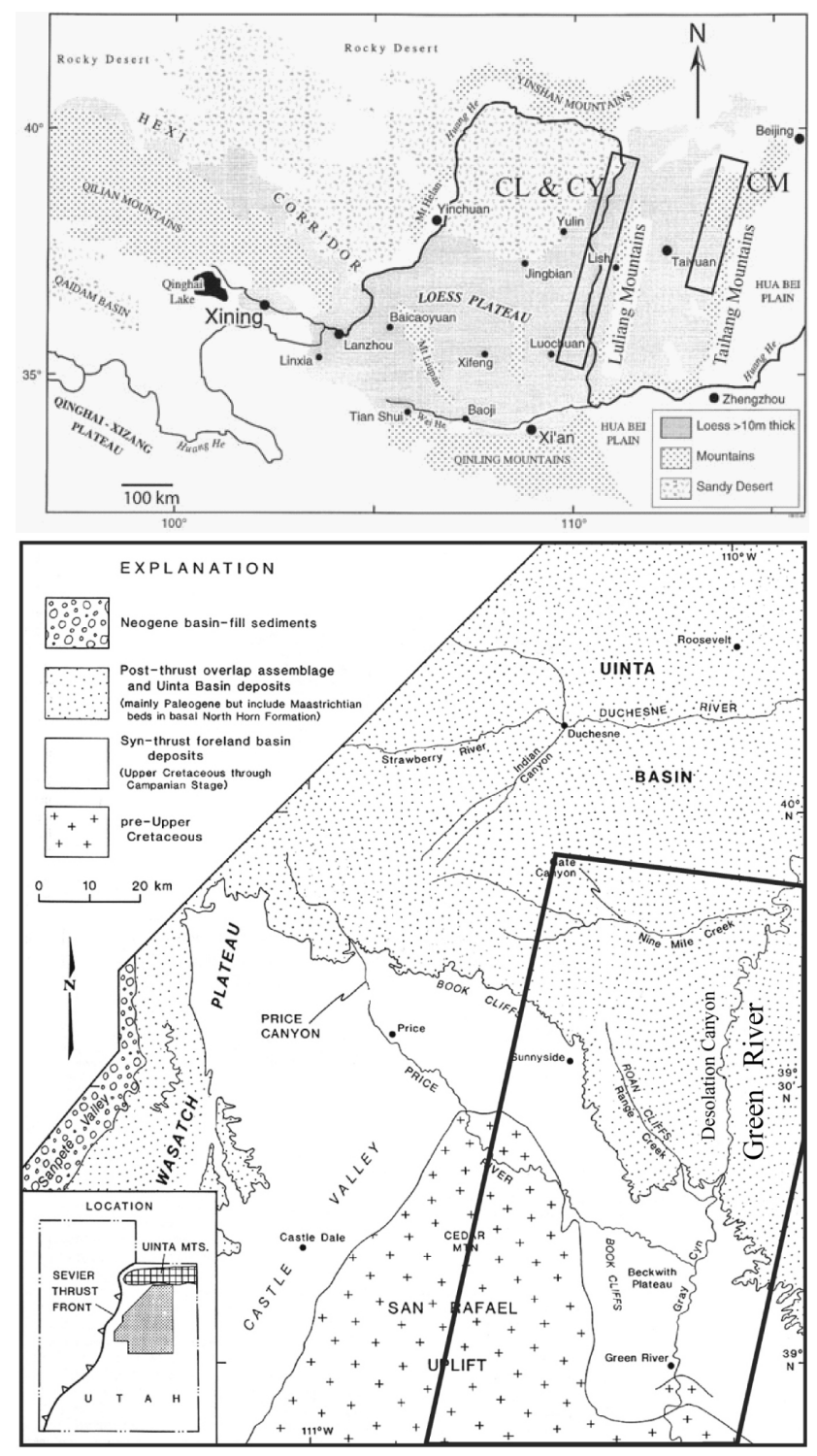

Fig. 1. (a) Location of selected regions in the Loess Plateau of China. The ASTER flight over the Yellow River (Huang He River) contains 8 scenes, overall representing $60 \mathrm{~km}$ by $485 \mathrm{~km}$; the CL and CY images were chosen in this section. The other ASTER flight is partly on the Taihang Mountains and is made of 5 scenes, overall representing $60 \mathrm{~km}$ by $304 \mathrm{~km}$; the CM images were taken from this area. The map is modified after Derbyshire (2001). (b) Location of the Utah data set (UT). The dark rectangle shows portion of the ASTER flight, which is composed of 3 scenes, and is $60 \mathrm{~km}$ by $184 \mathrm{~km}$. The map is modified after Dickinson et al. (1986).

\section{Isotropic scaling of the radiance fields}

\subsection{Method}

Investigation of the isotropic scaling properties of each image was performed with spectral analysis. The isotropic Fourier 


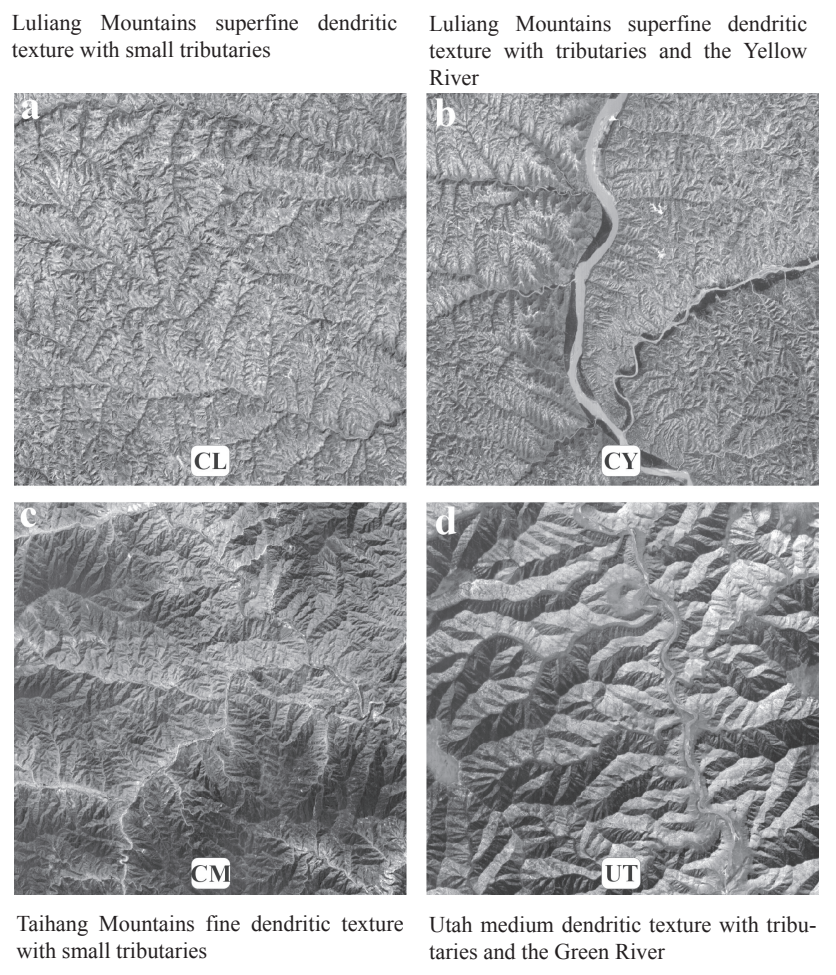

Fig. 2. Examples of the drainage pattern in each of the data groups. (a) the CL group, (b) the CY group (c) the CM group, (d) the UT group. Each image is 512 by 512 pixels and is $15.36 \times 15.36 \mathrm{~km}^{2}$.

power spectrum is a second order statistic and hence is only a single member of the hierarchy of exponents necessary to fully characterize multiscaling/multifractal fields. However, it is adequate for our present purposes; for studying the anisotropy. We do not attempt to characterize anisotropies of the other statistical moments, but this could be done, for example by considering the spectrum of various powers of the original field.

The spectral density $P(\underline{k})$ is defined as the ensembleaveraged, square modulus of the Fourier transform $\left.\tilde{I}(\underline{k})=\int e^{i k \underline{k}} \underline{x} I \underline{x}\right) d \underline{x}$ of the image $I(\underline{x})$ :

$$
P(\underline{k})=\left\langle|\tilde{I}(\underline{k})|^{2}\right\rangle
$$

where "<.>" denotes ensemble (statistical) averaging. Although the original real space structures may be highly elongated, $P(\underline{k})$ is an average over all of them independently of location; its variation with the direction of $k$ represents a kind of spatial and ensemble averaged anisotropy.

We can now define the (isotropic) spectrum $E(k)$ as the angle-integral of $P(\underline{k})$ :

$$
E(k)=\int_{\left|\underline{k^{\prime}}\right|=k} P\left(\underline{k^{\prime}}\right) d \underline{k^{\prime}} .
$$

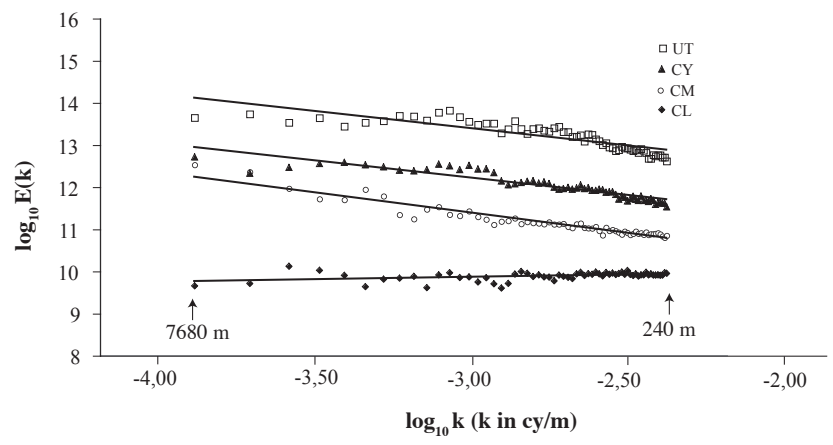

Fig. 3. Plot of $\log _{10} E(k)$ versus $\log _{10}(k)$ for selected images of each group; they are shifted (from each other by 1 ) for a better visualization. The $\beta$ values of these individual spectra are respectively from top to bottom: $0.82 ; 0.82 ; 0.96 ;-0.12$. See Table 1 for average $\beta$ values over the various sets.

(the notation indicates integration over all wave vectors of length $k=|\underline{k}|$, i.e. over all angles). If the scaling is isotropic we have:

$$
\begin{aligned}
& P(\underline{k}) \propto|\underline{k}|^{-s}=k^{-s} \\
& E(k) \propto k^{-\beta} \quad \beta=s-D+1
\end{aligned}
$$

where $\beta$ and $s$ are the scale invariant exponents; $D$ is the dimension of space ( $=2$ for images), and $k=|k|$ is the modulus of the wave vector ( $k$ is in cycles/meter or cycles/pixel). Below, we estimate $P(\underline{k})$ from a single realization (single image) since the different images we have are presumably not realizations of the same process (in particular do not have the same anisotropy parameters).

\subsection{Results}

The isotropic analyses demonstrate the presence of scale invariance of the TERRA ASTER visible radiance fields between $240 \mathrm{~m}$ ( 8 pixels) and $7680 \mathrm{~m}$ (256 pixels) corresponding to $\log _{10}(k)=-2.38, \log _{10}(k)=-3.89$ with $k$ in cycles/meter over the China and Utah regions. Representative individual image spectra of the four data sets $(\mathrm{CL}, \mathrm{CM}$, $\mathrm{CY}$ and UT) are presented in Fig. 3 (all spectra were calculated using Hanning windows). The isotropic scaling below $240 \mathrm{~m}$ was not considered satisfactory for the ensemble of data sets. As previously observed by other studies (Laferrière and Gaonac'h, 1999; Beaulieu and Gaonac'h, 2002), breaks in the isotropic scaling at higher resolution often occur when images are pre-processed (geometric/radiometric corrections). In the case of the TERRA ASTER data, vertical banding is an obvious artifact which affects the scaling at the highest resolutions. At the large scales, structures larger than half the linear size of each image are rare and thus don't provide reliable low wave number statistics; we thus omitted the $k=1$ statistics. When considering all images, the regionally averaged $\beta$ 's (Table 1 ) above $240 \mathrm{~m}$ show an increase 
Table 1. Average estimates of the isotropic scale invariant parameters of each data set with associated standard deviations indicating the image to image variation of the distribution of the parameters within each group of images. $\ln \left(l_{s}\right)$ is estimated with Eq. (14); the corresponding distance in meters is indicated in parentheses; $a^{2}$ has been estimated for each image using Eq. (21).

\begin{tabular}{ccccc}
\hline \multicolumn{5}{c}{ Estimates of the averages \pm Standard deviation } \\
\hline CL & CY & UT & CM \\
\hline$\beta$ & $0.09 \pm 0.15$ & $0.76 \pm 0.23$ & $0.85 \pm 0.20$ & $0.91 \pm 0.18$ \\
$\zeta_{1}$ & $-0.19 \pm 0.19$ & $0.24 \pm 0.12$ & $0.18 \pm 0.05$ & $-0.07 \pm 0.17$ \\
$\zeta_{0}$ & $1.94 \pm 1.29$ & $-0.94 \pm 0.80$ & $-0.18 \pm 0.31$ & $1.06 \pm 1.10$ \\
$\theta_{1}$ & $0.09 \pm 0.45$ & $0.55 \pm 0.82$ & $0.02 \pm 0.08$ & $-0.13 \pm 0.34$ \\
$\theta_{0}$ & $-0.05 \pm 1.13$ & $0.34 \pm 1.17$ & $0.25 \pm 0.37$ & $0.08 \pm 0.86$ \\
$\ln \left(l_{S}\right)$ & $6.89 \pm 5.45(980)$ & $3.00 \pm 2.44(20)$ & $0.68 \pm 1.47(2)$ & $9.73 \pm 6.43(17000)$ \\
$a^{2}$ & $0.01 \pm 0.03$ & $0.02 \pm 0.02$ & $0.01 \pm 0.01$ & $0.00 \pm 0.003$ \\
\hline
\end{tabular}

from $\mathrm{CL}(\beta=0.09)$ to $\mathrm{CM}(\beta=0.91)$. CL images exhibit a distinct scaling characterization compared to the others $(\mathrm{CM}$, $\mathrm{CY}$ and UT) with an extremely low $\beta$ value $(\bar{\beta}=0.09)$ indicating that there are very few large structures compared to smaller ones in this superfine drainage area of the China Loess Plateau (CY). On the other hand, the other three image groups exhibit higher $\beta$ 's (CY, CM and UT, $\bar{\beta}=0.76,0.91$ and 0.85 , respectively) reflecting the increased dominance of large structures in the images compared to small ones but in a scale invariant relationship. Hence, while isotropic scaling prevails over the four distinct drainage groups it does not statistically distinguish the four different areas, which obviously reveal distinct patterns (Fig. 2).

\subsection{Discussion}

The relatively low $\beta$ values of the superfine textural CL images may be explained by the homogeneous thick deposit of loess where many small source tributaries have developed, creating a very high-density low order drainage system (Fig. 2a). The other three data sets (CY, CM, UT), have larger $\beta$ values, reflecting the existence of more dominant large structures: a) the higher $\mathrm{CY} \beta$ values may be explained by the presence of the large and developed Yellow River valley (Fig. 2b) cutting through the loess (producing superfine texture); b) the fine textural CM images, which present a pattern of dendritic source tributaries (as in CL images) could reflect with their higher $\beta$ 's the combined effect of loess deposits thinner and thinner to the east and shallow underlying crystalline hard rocks, which may partially impose some structural control on the regional stream network (high $\beta$ 's indicate "rough" images of images while low $\beta$ 's smoother ones). Hence, the thicker the loess is at the tributary source (CL compared to $\mathrm{CM}$ ), the lower the $\beta$ values of the radiance fields; c) finally the medium textural UT images have distinct $\beta$ values from the $\mathrm{CL}$ images, easily explained by the large sedimentary structures present in the Utah images including the presence of the large Green River. Similar obser- vations of distinct $\beta$ values, from differentially eroded portions of a river network located on the Ethiopian Plateau, were found by Beaulieu and Gaonac'h (2002). Maître and Pinciroli (1999) also suggested a possible link between the fractal dimension and various geomorphologic aspects of the surface, such as soil composition and friability, where low fractal dimensions would be associated with friable quick erosional environments and high values with dissected hard rock settings. However, Fourier and multiscaling analyses better characterize scale invariant fields since fractal geometrical dimensions will depend on the radiance or altitude on which they are defined and will generally not produce unique values.

\section{Anisotropy of the radiance fields}

\subsection{Generalized scale invariance}

If we "zoom" into a self-similar (isotropic and scaling) system, the magnified structures are on average of the same type as the unmagnified ones. In order to obtain the same simple relation between large and small structures in a scaling but anisotropic system, the zoom must be accompanied by a compression and/or rotation as a function of scale. To handle anisotropy in scaling system the generalized scale invariance (GSI) framework is needed (Schertzer and Lovejoy, 1985). Anisotropic scaling can be described by a scale-changing operator $T_{\lambda}$ which reduces scale by a factor $\lambda$. The operator $T_{\lambda}$ may be written as:

$T_{\lambda}=\lambda^{-\mathbf{G}}$

where $\mathbf{G}$ is the generator that takes the form of the identity matrix (1) for the isotropic (self-similar) case. A second element is a unit "ball" which defines all the unit vectors. From the unit ball $B_{1}$ we can generate a family of balls by $B_{\lambda}=T_{\lambda} B_{1}$ i.e., the scale of all the non-unit vectors is defined by applying $T_{\lambda}$ to $B_{1}$. The scale function $\|\underline{x}\|$ specifies 


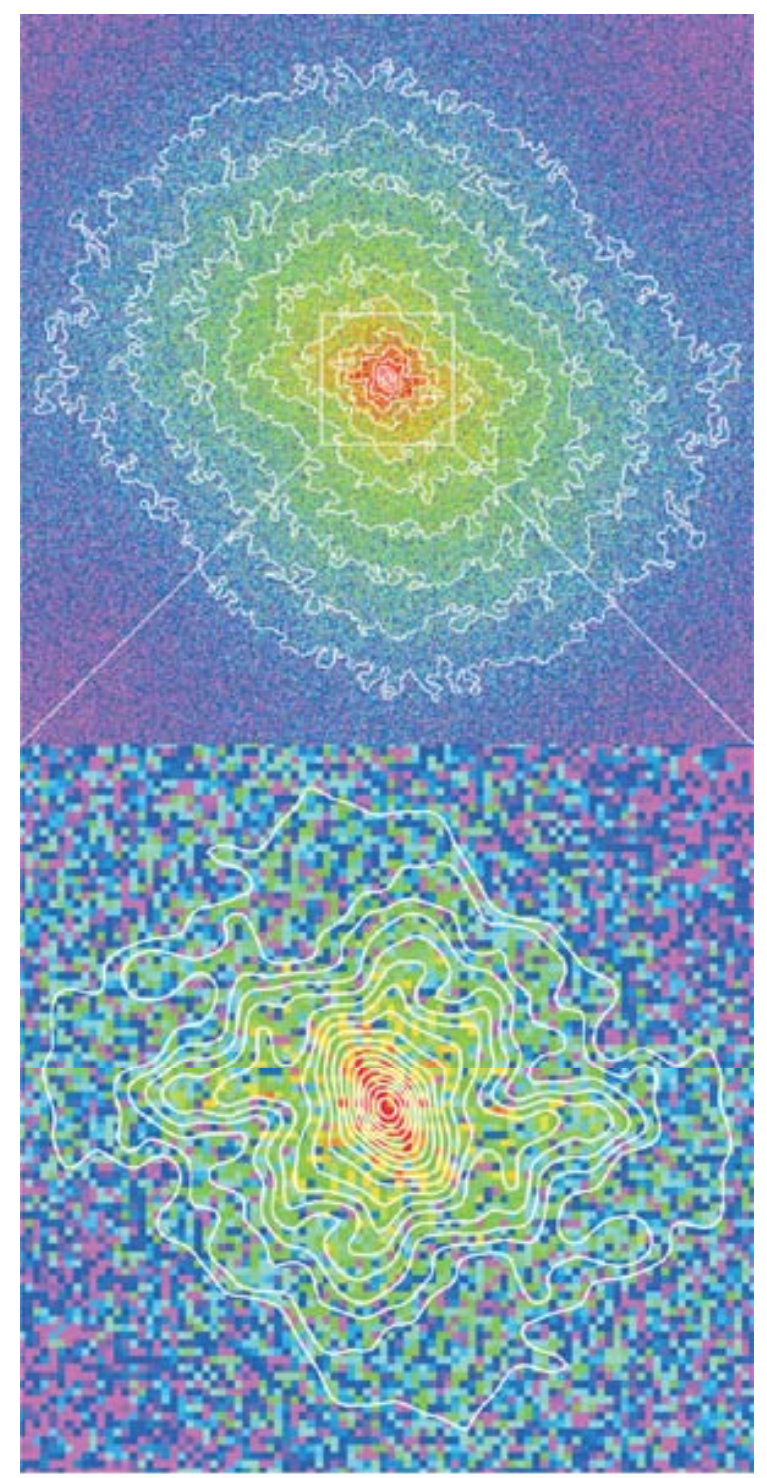

Fig. 4. Example of the fitted contours of $P(\underline{k})$ of a 512 by 512 pixel $\mathrm{CL}$ image. The bottom image (b) is a magnification of the small real space square in the top image (a). In $P(\underline{k})$, the small structures are depicted by the large contours, and the large ones by the small contours; they are also orthogonal to the real space structures.

the size of a vector $\underline{x}$; it must satisfy the scale function equation $\left\|T_{\lambda} \underline{x}\right\|=\lambda^{-1}\|\underline{x}\|$. In words, this means that the size of a reduced scale vector $\left(T_{\lambda} \underline{x}\right)$ is $\lambda$ times smaller than the size of the original (large scale) vector $x$. When $\mathbf{G}=\mathbf{1}$, a simple solution of the scale equation is $\|\underline{x}\|=|\underline{x}|$, i.e. the scale can be taken as the usual vector norm (length). In an anisotropic scaling system, the size (scale) of a vector $\underline{x}$ is thus defined by $\| \underline{x}||$ rather than the usual norm $|\underline{x}|$. If anisotropy is present in a system, there will generally be one scale $\left(l_{s}\right)$ at which $\left\|\left(l_{s}, 0\right)\right\|=\left\|\left(0, l_{s}\right)\right\|$ (however, see below for the apparently exceptional case of rotation dominance when there can be infinitely many or none); $l_{s}$ is called the "sphero-scale" since the corresponding structures will typically be "roundish". Although it is not strictly necessary, in many cases of weak scaling anisotropy such as those studied here, structures are fairly roundish (see for example Fig. 4a). If $B_{1}$ is a circle, since $T_{\lambda}$ is a linear operator, it will transform the circle to another quadratic form: the $B_{\lambda}$ are ellipses. Since the images are $2-\mathrm{D}, \mathbf{G}$ is a $2 \times 2$ matrix which can be conveniently represented by a linear combination of basic quaternion-like elements (Schertzer and Lovejoy, 1985; Lovejoy and Schertzer, 1985):

$\mathbf{G}=d \mathbf{1}+c \mathbf{K}+f \mathbf{J}+e \mathbf{I}$

where

$\mathbf{1}=\left(\begin{array}{ll}1 & 0 \\ 0 & 1\end{array}\right), \mathbf{K}=\left(\begin{array}{cc}1 & 0 \\ 0 & -1\end{array}\right), \mathbf{J}=\left(\begin{array}{ll}0 & 1 \\ 1 & 0\end{array}\right), \mathbf{I}=\left(\begin{array}{cc}0 & -1 \\ 1 & 0\end{array}\right)$

or:

$\mathbf{G}=\left(\begin{array}{ll}d+c & f-e \\ f+e & d-c\end{array}\right)$

where $c$ and $f$ are associated with stratification, $e$ with rotation and $d$ with an overall contraction of the system. The eigenvalues of $\mathbf{G}$ are $d+a, d-a$ where:

$a^{2}=c^{2}+f^{2}-e^{2}$

so that we see that the general qualitative behavior of linear GSI is determined by $a$. Two different cases can be described. When $a^{2}>0$, the stratification of the structures is dominant with rotation never exceeding $\pi / 2$. When on the contrary, $a^{2}<0$, the rotation is dominant, the balls, or ellipses rotate as the scale changes through an infinite angle of rotation as $\lambda \rightarrow \infty$. When rotation and stratification both act, they can sometimes give $a^{2}$ values near zero, since they counteract each other.

\subsection{The "Differential Anisotropy Scaling" technique}

The empirical estimate of GSI parameters is difficult; Lewis et al. (1999) proposed the "scale invariant generator, SIG" technique that involves a nonlinear regression in a fourdimensional parameter space on $P(\underline{k})$. The difficulty with the SIG method is that although it was tested on ideal cases (i.e. simulations) in many empirical fields the parameter space turned out to be too large for convergence to the global error function minimum. As an alternative, in the present study, contours are fitted to $P(\underline{k})$, and the GSI parameters are then extracted from contours (Fig. 4). This method is in many ways similar to that of Pflug et al. (1993) except that we use a weak anisotropy approximation which (when valid) has several advantages. In particular its parameters are estimated by two linear regressions so that estimates are more robust. In addition the parameters have a straightforward interpretation whether or not the system is scaling. To each contour in an image (in $P(\underline{k})$ ), ellipses are fitted and the major 
axis $A$, the minor axis $B$ and the angle of orientation $\theta$ (between the major axis and the horizontal axis) are estimated. We assume both linear GSI ( $\mathbf{G}$ is a matrix) and also weak anisotropy (i.e. near elliptical balls; see the exact characterization below). The simplest way to obtain the basic equation of the "Differential Anisotropic Scaling" technique is to start with the characterization of the transformation of ellipses under the action of $\lambda^{-\mathbf{G}}$. Since $\lambda^{-\mathbf{G}}$ is a linear transformation, the method maps ellipses onto other ellipses; it suffices to know the orientation and ellipticity of the ellipses as functions of scale. We follow the results of Pflug et al. (1993); who showed that the major and minor axes of an ellipse ( $A$ and $B$ ), for each contour the ellipticity can be characterized by:

$\zeta(l)=\sqrt{\sqrt{\frac{A(l)}{B(l)}}-\sqrt{\frac{B(l)}{A(l)}}}$

Since by definition $B(l)<A(l)>0$. Note that $\varepsilon=A / B-1$ is the more usual "ellipticity"; we have $\zeta^{2}=\varepsilon / \sqrt{\varepsilon+1}$. When there exists a roughly isotropic scale (or sphero-scale) in the image, the ellipticity of its ensemble of structures may be related to the anisotropic scaling GSI parameters via:

$\zeta^{2}(u)=2 r \frac{\sinh ^{2}(a u)}{a} ; a^{2}>0$, stratification dominance (10a)

$\zeta^{2}(u)=2 r \frac{\sin ^{2}(|a| u)}{|a|} ; a^{2}<0$, rotation dominance

where $u=\ln (\lambda), \lambda=l / l_{s}, l$ is the size or scale of the structure ( $k=2 \pi / l$ is the equivalent in Fourier space) and $l_{s}$ is the sphero-scale. $l$ is estimated as the square root of the area of the corresponding ellipses; this corresponds to using $d=1$ (see Lewis et al., 1999). We have introduced $r=\sqrt{c^{2}+f^{2}}$; $c=r \cos \varphi$ and $f=r \sin \varphi, \varphi$ being an angle in the (c, f) space. Using the approximation $\sinh x \approx x$ and using $u=\ln (l)-\ln \left(l_{s}\right)$ we obtain:

$\zeta(u)=|u| \sqrt{2 r|a|}+O(a u)^{3}$

where "O" means "of order". To lowest order this is equivalent to:

$\zeta(l) \approx\left|\ln (l)-\ln \left(l_{s}\right)\right| \sqrt{2 r|a|}$

The positive root is taken since $\zeta>0$; the approximation Eq. (11) is valid for small $|a u|$. Equation (11b) can also be expressed as:

$\zeta(l) \approx \zeta_{0}+\zeta_{1} \ln (l)$

with:

$\zeta_{0}=-\ln \left(l_{s}\right) \sqrt{2 r|a|}$ and $\zeta_{1}=\sqrt{2 r|a|}$ for $l>l_{s}$

$\zeta_{0}=\ln \left(l_{s}\right) \sqrt{2 r|a|}$ and $\zeta_{1}=-\sqrt{2 r|a|}$ for $l<l_{s}$
This expansion is valid as long as the linear term of the Taylor expansion dominates the cubic one, i.e.:

$u \ll \frac{\sqrt{6}}{|a|}$

Pecknold et al's. (1997) analysis of various geophysical fields showed that $|a|$ is frequently of the order of 0.1 and usually $<0.2$; this implies that the following is applicable if we are within a factor of $\sim 10^{5}$ of the sphero-scale (i.e., $\left.l / l_{s} \leq e^{\sqrt{6} / 0.2}\right) . \zeta(l)$ can be plotted against $\ln (l)$. From the data and Eq. (12), the slope $\zeta_{1}$ and the intercept $\zeta_{0}$ (i.e. the ellipticity at scale $l=l_{s}$ or $\ln \left(l / l_{s}\right)=0$ ) can be retrieved. In this case, the parameter $\zeta_{1}$ represents the differential anisotropic variation from scale to scale of the system. The sphero-scale $l_{s}$ is estimated as:

$l_{s}=e^{-\zeta_{0} / \zeta_{1}}$

In order to get more information about the matrix generator G (see Eq. 7), we can consider the variation with scale of the angle of the axis of the ellipse with respect to the horizontal. Pflug et al. (1993) expressed it as:

$\theta(u)=\frac{1}{2} \tan ^{-1}\left(\frac{f}{c}\right)-\frac{1}{2} \tan ^{-1}\left(\frac{e}{a} \tanh (a u)\right)$

(valid for a real or imaginary). Expanding this expression in au gives:

$\theta(u)=\frac{1}{2} \tan ^{-1}\left(\frac{f}{c}\right)-\frac{1}{2} e u+O(a u)^{3}$

This approximation $\theta(u) \approx \frac{1}{2} \tan ^{-1}\left(\frac{f}{c}\right)-\frac{1}{2} e u$ is valid as long as:

$|u| \ll \frac{\sqrt{3}}{r}$

which is very close to the previous condition (Eq. 13). Then, according to the above, we can represent the angle variation between the major axis and the horizontal as:

$\theta(l) \approx \theta_{0}+\theta_{1} \ln (l)$

where $\theta_{0}=\frac{\varphi}{2}+\frac{e}{2} \ln \left(l_{s}\right)$ and $\theta_{1}=-\frac{e}{2}, \varphi=\tan ^{-1}\left(\frac{f}{c}\right) . e$ is used in real space, $-e$ in Fourier space (this is because the $\mathbf{G}$ in Fourier space is the transpose of the real space G). From Eqs. (8), (12) and the parameter $e$ retrieved from $\theta_{0}$, we can rewrite $\zeta_{0}$ and $\zeta_{1}$ :

$\zeta_{0}=-\ln \left(l_{s}\right) \sqrt{2 r \sqrt{\left|r^{2}-e^{2}\right|}} \quad l>l_{s}$

$\zeta_{0}=\ln \left(l_{s}\right) \sqrt{2 r \sqrt{\left|r^{2}-e^{2}\right|}} \quad l<l_{s}$

and

$\zeta_{1}=\sqrt{2 r \sqrt{\left|r^{2}-e^{2}\right|}} l>l_{s}$ 
(a)

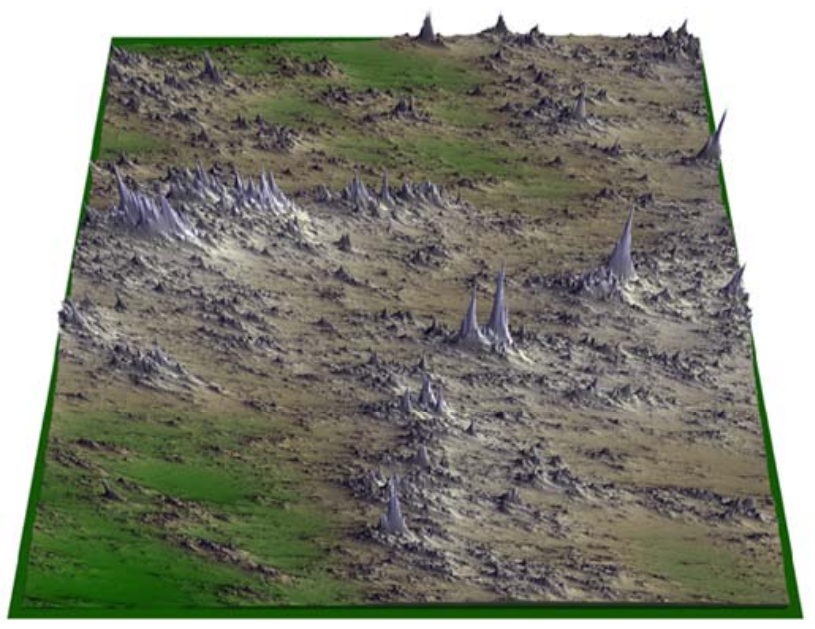

(b)

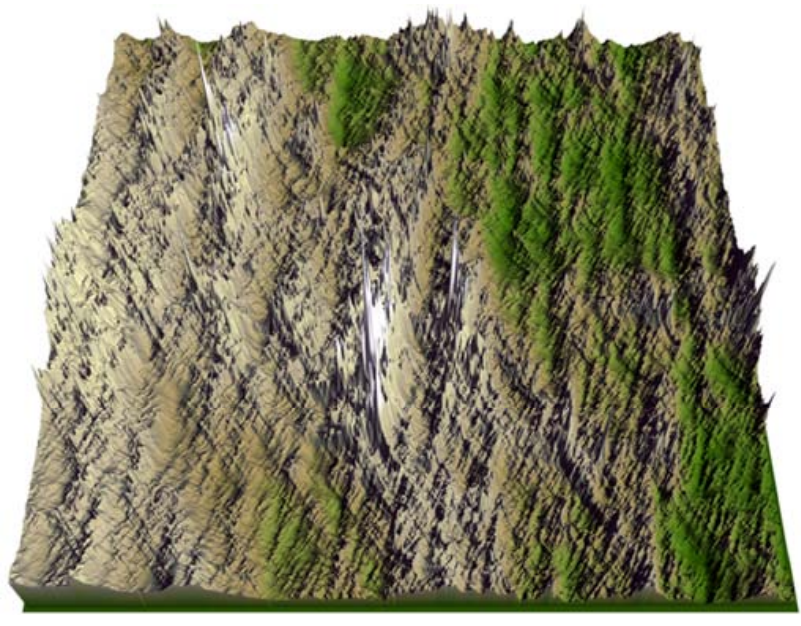

Fig. 5. Example of two anisotropic multifractal simulations $(512 \times 512$ pixels) with identical multifractal parameters $\alpha=1.90$, $C_{1}=0.12$ and $H=0.7$ These are the observed values for the topography (see Gagnon et al., 2006). (a) Anisotropic theoretical parameters are $d=1, c=0.2, e=0.1, f=0, l_{S}=0.5$ and $a=0.173$. (b) Same except for $l_{s}=4096$.

$$
\zeta_{1}=-\sqrt{2 r \sqrt{\left|r^{2}-e^{2}\right|}} \quad l<l_{s}
$$

Since the sign of $\zeta_{1}$ changes at the sphero-scale, if the latter is within the observable scale range, this fact can be used to identify it. Finally, since $a^{2}=r^{2}-e^{2}$, Eq. (20) can be solved for $r^{2}$ :

$r^{2}=\frac{e^{2}+\sqrt{e^{4}+\zeta_{1}^{4}}}{2}, a^{2}>0$

and

$r^{2}=\frac{e^{2} \pm \sqrt{e^{4}-\zeta_{1}^{4}}}{2}, a^{2}<0$

hence, when $\left|\zeta_{1}\right|>|e|$, we must have a stratification dominant regime $\left(a^{2}>0\right)$ and a unique solution for $r^{2}$. However,

\section{Simulation A}

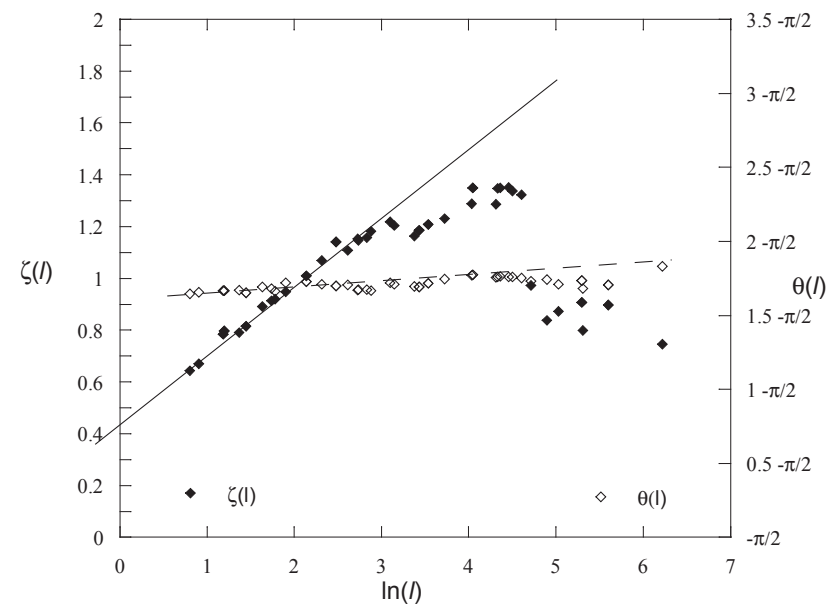

Simulation B

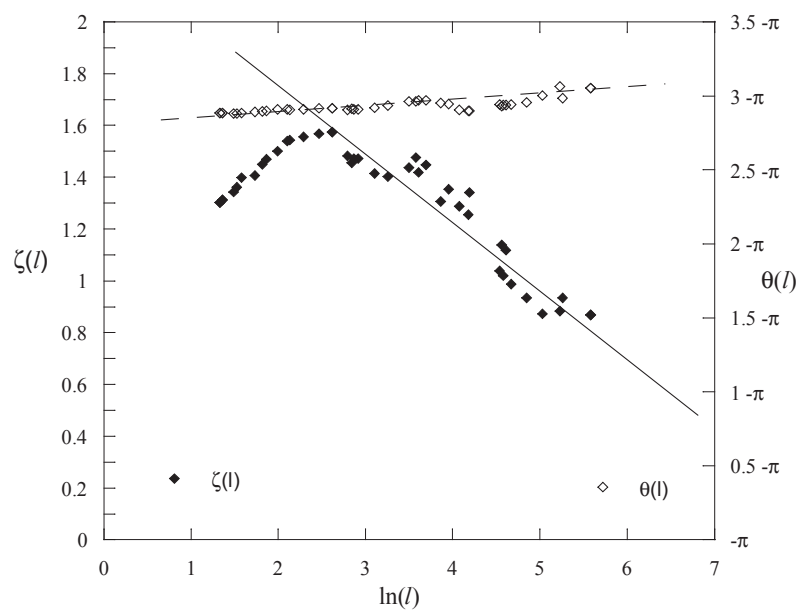

Fig. 6. Plots of $\zeta(l)$ (filled symbols) and $\theta(l)$ (open symbols) as functions of $\ln (l)-l$ in pixels - for simulations $5 \mathrm{a}$ and $5 \mathrm{~b}$. These two simulations differ only in $l_{s}$. The black lines are the theoretical $\zeta(l)$ retrieved from Eq. (12) plotted on the data analysis of the simulations $5 \mathrm{a}$ and $5 \mathrm{~b}$. The dash lines are the theoretical $\theta(l)$ retrieved from Eq. (18). See explanations in text.

when $\left|\zeta_{1}\right|<|e|$, we have two additional possible solutions for $a^{2}<0$. In this case, to establish the sign of $a^{2}$ we must consider the third order term of the expansion of the expression of $\zeta$ (Eq. 11a).

\subsection{Tests of the Differential Anisotropy Scaling technique} on numerical simulations

This analytical method was applied on anisotropic multifractal simulations $(512 \times 512$ pixels $)$ such as the fields in Fig. 5. Results are presented for two simulations, which are analyzed through their spectral densities $P(\underline{k})$. We can observe (Fig. 6) the log-linear relationship implied by the GSI 
Table 2. Theoretical and estimated anisotropic values of the two simulated $5 \mathrm{a}$ and $5 \mathrm{~b}$ fields. Estimated $\zeta_{1}, \zeta_{0}, \theta_{1}, \theta_{0}$ values are retrieved from Fig. 6 following the ln-linear equations Eq. (12) and Eq. (18). Estimated $l_{s}$ is calculated from Eq. (14). Theoretical $\zeta_{1}, \zeta_{0}, \theta_{1}, \theta_{0}$ are calculated from simulations values (using the negative of $e$ for comparison with Fourier estimates). $\theta_{0}$ has been chosen to lie between $-\frac{\pi}{2}$ and $\frac{\pi}{2}$ as well as to take into account the sign of $\zeta_{1}$ (as discussed in the text).

\begin{tabular}{|c|c|c|c|c|}
\hline \multicolumn{5}{|c|}{ Theoretical and numerically estimated anisotropic values of simulations $5 \mathrm{a}$ and $5 \mathrm{~b}$} \\
\hline & Theory $5 \mathrm{a}$ & $\begin{array}{l}\text { Numerically } \\
\text { estimated } 5 a\end{array}$ & Theory $5 b$ & $\begin{array}{l}\text { Numerically } \\
\text { estimated } 5 b\end{array}$ \\
\hline$\zeta_{1}$ & 0.263 & 0.196 & -0.263 & -0.253 \\
\hline$\zeta_{0}$ & 0.182 & 0.561 & 2.188 & 2.256 \\
\hline$\theta_{1}$ & 0.05 & 0.026 & 0.05 & 0.031 \\
\hline$\theta_{0}$ & 0.035 & 0.061 & -0.416 & -0.316 \\
\hline$l_{s}$ & 0.50 & 0.057 & 4096 & 7458 \\
\hline$a$ & 0.173 & 0.133 & 0.173 & 0.170 \\
\hline
\end{tabular}

model (Schertzer and Lovejoy, 1985; Lovejoy and Schertzer, $1985)$ between $\zeta(l)$ and $\ln (l)$ (Eq. 12; filled symbols in Fig. 6) and between $\theta(l)$ and $\ln (l)$ (Eq. 18); open symbols in Fig. 6); we have passed from Fourier to real space using $l=2 \pi /|\underline{k}|$. A summary of the theoretical values obtained from the two simulations and estimated values retrieved from their analyzed image contours is presented in Table 2 . We observed that $\zeta_{1}$ as well as $\theta_{1}$ slopes of the theoretical fits (straight lines) are reasonably close to the estimated $\zeta_{1}$ and $\theta_{1}$ values for both simulations.

The values of $\zeta_{0}$ are determined by the Fourier space sphero-scale $\left(k_{s}\right)$. Since there is no simple relation between Fourier shapes and real space shapes, the use of $2 \pi / k_{s}$ to estimate $l_{s}$ is only approximately valid. It is therefore not surprising that the $l_{s}$ used to produce the simulation and $2 \pi / k_{S}$ estimated from linear regressions on the Fourier estimated $\zeta$ are not identical. We therefore do not expect excellent agreement between Fourier and real space $\zeta_{0}$ parameters. However $\zeta_{1}, \theta_{0}, \theta_{1}$ do not have this problem; we therefore expect the only differences between real and Fourier space to be the sign of $e$ and hence $\theta_{1}$. Schematically we have: $\zeta_{0 r} \approx \zeta_{0 f}$; $\zeta_{1 r}=\zeta_{1 f} ; \theta_{0 r}=\theta_{0 f} ; \theta_{1 r}=-\theta_{1 f}$, where " $r$ " indicates "real" space and " $f$ " indicates "Fourier" space. The only additional complication is in the estimation of the orientation angle $\theta$. First, we note that axes of ellipses are only defined to within $\pi$; for convenience we take $-\pi / 2<\theta<\pi / 2$. Next, the major and minor axes change places at the sphero-scale: the reduced scale image below the sphero-scale of a major axis is a minor axis and visa versa. This has the effect of adding $\pi / 2$ to $\theta_{0}$ below the sphero-scale (see Table 2).

Table 2 shows the comparison of the theoretical and estimated parameters for the two simulations shown in Fig. 5 with different $l_{s}$. This leads to differences in the signs of the slopes $\zeta_{1}$ : positive and negative respectively on the plot of $\zeta(l)$ versus $\ln (l)=\ln (2 \pi / k)$. According to the above, we must therefore correct the $\theta_{0}$ values by $\pi / 2$ in the former, but not in the latter case. From the table and the figure, we see that when this is done, the method does a reasonable job of recovering the theoretical parameters. We conclude that our method is adequate for the characterization of not too anisotropic simulated fields ( $\operatorname{small} a \ln \left(l / l_{s}\right)$ ). We will below use estimates of all these parameters for comparison between the different drainage area groups.

\subsection{Empirical results}

Estimates of the $\zeta_{1}$ parameters (anisotropic variation with scale) for all remotely sensed images was done using the $P(\underline{k})$ contours as described above. Data points below $240 \mathrm{~m}$ were considered for extracting statistical properties since the "Differential Anisotropic Scaling" analyses did not demonstrate breaks in the $\zeta(l)$ versus $\ln (l)$ behavior over the range 120 to $240 \mathrm{~m}$. Although the isotropic scaling was not so well displayed between $120 \mathrm{~m}$ and $240 \mathrm{~m}$, the anisotropic scaling is not as much affected and provides useful information. Indeed the new method exhibiting anisotropic scaling does not necessarily imply a well defined isotropic scaling. Hence, we decided to keep all the data above $120 \mathrm{~m}$ resolution to improve the statistics. The estimates were then made up to the largest available scale. The range of scales over which the anisotropic scaling was estimated varies from one image to another while the isotropic scaling was determined over $240 \mathrm{~m}$ to half the image size $(7680 \mathrm{~m})$. The anisotropic scaling was revealed by the present "Differential Anisotropic Scaling" technique over a range of scales of 100-1350 m for the UT set, 375-3100 $\mathrm{m}$ for the CM group, 260-2000 $\mathrm{m}$ for the CY group and finally $225-2750 \mathrm{~m}$ for the CL set. Figure 7 gives an example of anisotropic scaling properties of two UT and CL images. The differential anisotropy present in both images is exhibited by $\zeta_{1}$ with opposite signs reflecting the fact that the Utah structures are increasingly stretched at larger scales while structures from the superfine textural dendritic drainage area of the China Loess Plateau (CL) are on the contrary increasingly roundish, a tendency reflected 

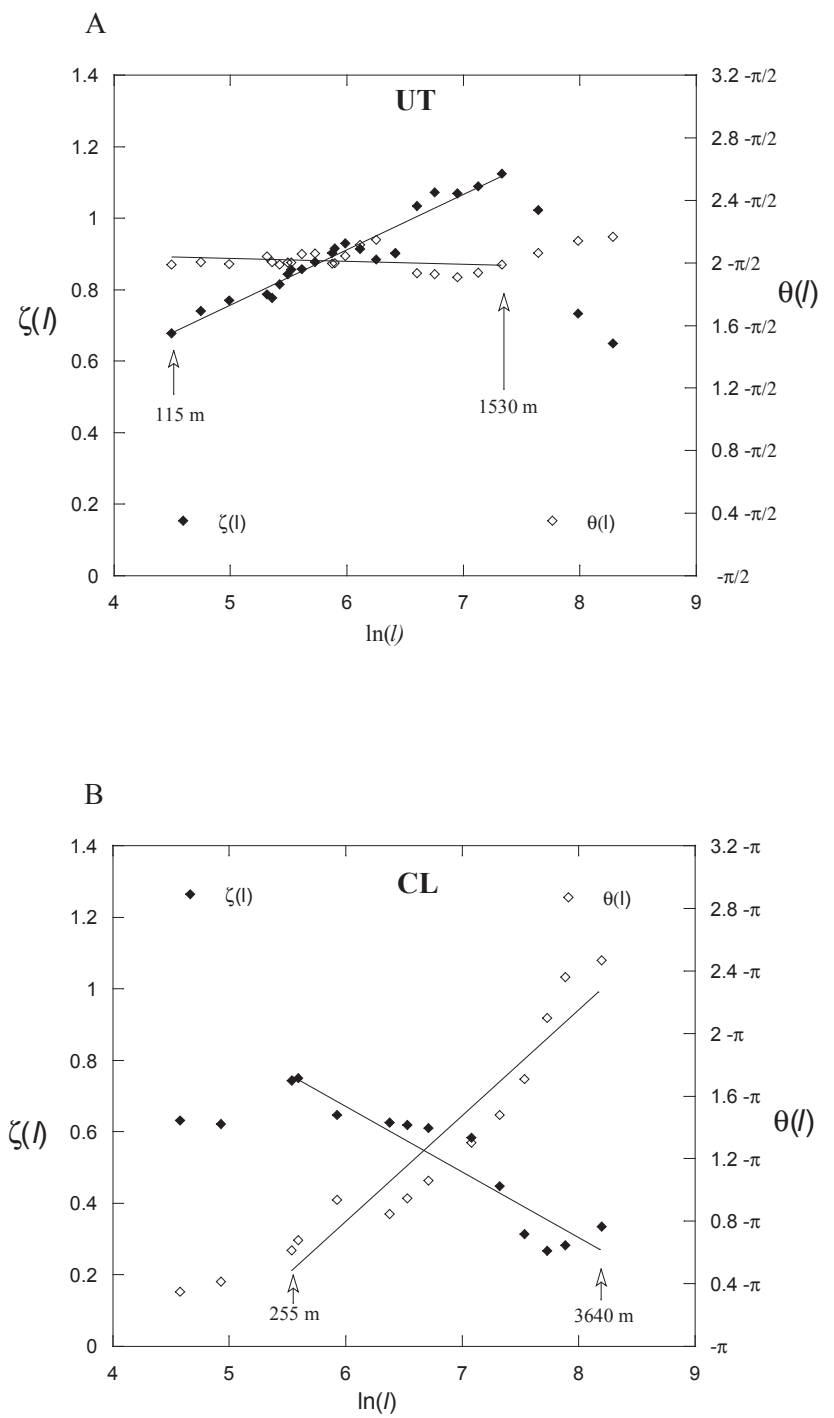

Fig. 7. Examples of $\zeta(l)$ (filled symbols) and $\theta(l)$ (open symbols) as functions of $\ln (l)-l$ in meters - on a UT sub-image (A) and on a CL sub-image (B). The lines were fit between the indicated scales (115 m-1530 m for UT; 255 m- $3640 \mathrm{~m}$ for CL).

by negative $\zeta_{1}$ values. When considering the rotation of structures with scale, the Utah structures exhibit a lower rate of rotation $\left(\theta_{1}\right.$ has a lower value) relative to the China CL image. The tendencies are confirmed by the averages (Table 1).

$\zeta_{1}$ and $\theta_{1}$ characterize the rates at which structures are respectively stretched and rotated with scale. In Figs. 8a, c we plot them against the isotropic spectral exponent $\beta$ in order to discriminate the very high density (superfine) dendritic China CL data set $(\bar{\beta} \approx 0.09)$ from the other sets (CY, CM and $\mathrm{UT} ; \bar{\beta}>0.70$ ). The parameter $\zeta_{1}$ separates the groups well, especially when considering the mean of each group (larger symbols), the CL and CM small tributaries groups $\left(\zeta_{1}<0\right)$ from the CY and UT groups $\left(\zeta_{1}>0\right)$ where larger distribu- taries are present. The $\theta_{1}$ values of all the data are confined between -1 and 1 , except for one point (Fig. 8c). $\theta_{1}$ doesn't improve classification with respect to $\zeta_{1}$; neither do $\zeta_{0}, \theta_{0}$ and $l_{s}$ (Figs. $8 \mathrm{~b}, \mathrm{~d}, \mathrm{e}$ ). Note that $\theta_{0}$ is the only parameter which depends on the absolute orientation of structures, it reflects preferential fixed directions.

Using group averages of $\zeta_{1}$ and $\zeta_{0}$ for respectively CL, CM, CY and UT, we extrapolated - following Eq. (12) - the ellipticity parameter $\zeta(l)$ estimated within the observed range of scales (limited by dashed lines in Fig. 9a). This reveals a range $(6<\ln (l)<8)$ where the UT group may be distinguished from the others (see arrow in Fig. 9a). For $\ln (l)>6$, the UT structures display more anisotropic characteristics. We thus decided to examine the absolute (single scale) anisotropy of the images for $\ln (l)$ above 6 and chose $960 \mathrm{~m}$ corresponding to $1 / 16$ of the image size (any other scale with $\ln (l)>6$ could have equally well been chosen). The plot of the anisotropy $\zeta(l)$ at this reference scale of $960 \mathrm{~m}-\zeta(960 \mathrm{~m})$ - versus the $\beta$ values indeed demonstrated a good discrimination between the UT group on one hand and the China CM, CL and CY groups on the other hand (Fig. 9b). Medium to large Utah structures show a lot of aligned, elongated structures (Fig. 2d) compared to other data sets, a result predicted by the anisotropic scaling properties of the data.

The parameter $a^{2}$ is a measure of the overall differential anisotropy of a system, including the stratification/contraction and rotation of the structures $\left(a^{2}=c^{2}+f^{2}-e^{2}\right)$. The $a^{2}$ values range between -0.01 and 0.10. While our "Differential Anisotropic Scaling" technique applies to structures and fields that are not too anisotropic (Eqs. 12 and 18), the observed small $a u$ values around 0 are consistent with this. The estimated spheroscales $l_{s}(\zeta(l)=0)$ are in general beyond the observed range of scales for the CL and CM groups because of the negative $\zeta_{1}$ values of these images, whereas the CY and UT groups show $l_{s}$ values around 20 and 2 meters, respectively also generally outside the observed range of scales (see Table 1).

\subsection{Discussion}

The analysis of differential anisotropy over the two different drainage areas proved to give insightful quantitative estimates of the satellite images. CL and CM groups are characterized by $\zeta_{1}<0$ reflecting that patterns exhibit less stratification at larger compared to smaller scales (Fig. 8a): i) in the CL group, the images show weak large structures due to the presence of a superfine dendritic texture of the region where small source tributaries are uniformly widespread all over the space; ii) in the CM images, large structures are present. We suggest they are the result of a thinner loess and a harder basement. Their spatial distribution however reflects the dominance of larger tributaries and valleys randomly distributing the water over the China Plateau revealing less overall statistical anisotropy on the images at larger scales. On the other hand, CY and UT images exhibit higher 

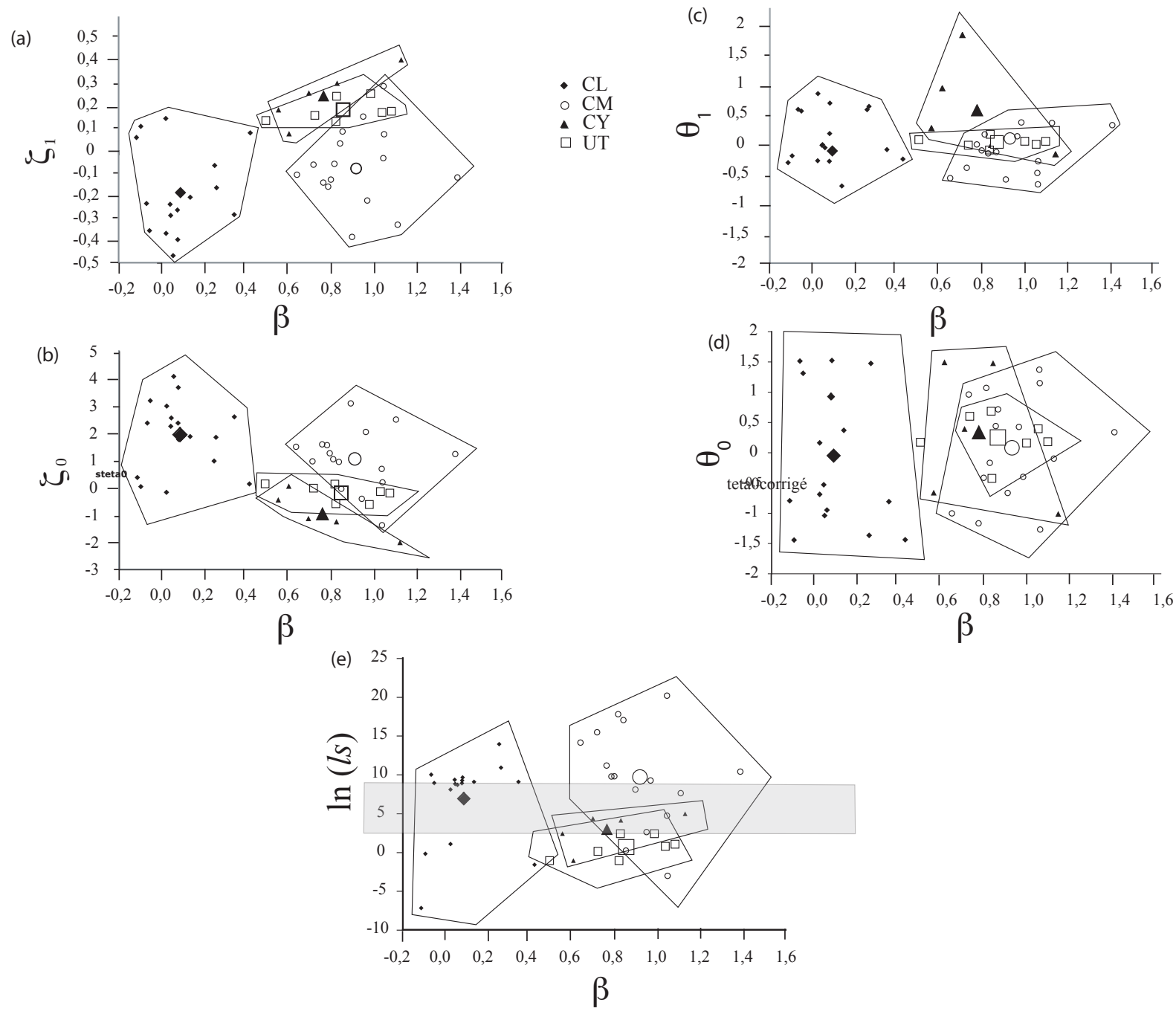

Fig. 8. Plots of anisotropic parameters versus the $\beta$ values estimated from $P(\underline{k})$ for the different groups. Symbols indicate same group in each plot. The large symbols represent the estimates of average values of each field. (a) $\zeta_{1} ;$ (b) $\zeta_{0} ;$ (c) $\theta_{1}$; (d) $\theta_{0}$ (in radians); (e) $\ln (l s)\left(l_{s}\right.$ in meters). The grey range in (d) indicates the range of image scales analyzed.

anisotropy at increasing scales $\left(\zeta_{1}>0\right.$; Fig. 8a). In the CY case, a major river, the Huang He (Yellow River), is present, creating a large very well oriented valley, hence drastically affecting the stratification at larger scales. On the UT images large rivers also have created deep elongated large valleys. Within these two groups of images involving large valleys (CY and UT), $\beta$ and $\zeta_{1}$ do not permit any distinctions between regions nor do the other parameters $\zeta_{0}, \theta_{1}, \theta_{0}, l_{s}$. The anisotropy at $960 \mathrm{~m}$ helps to differentiate the Utah from the three Chinese areas (Fig. 9b). As predicted by the linear $\zeta(l)$ obtained by using the region averaged $\zeta_{0}, \zeta_{1}$ parameters, all China groups $\zeta(960 \mathrm{~m})$ exhibit similar values $(\mathrm{CL}=0.72$, $\mathrm{CY}=0.74$ and $\mathrm{CM}=0.56$ ) explained as being related to the same drainage area (Yellow River system) developed in a peculiar geological environment while the Utah shows a higher $\zeta(960 \mathrm{~m})$ value (UT=1.06) reflecting its higher stratification of structures at $960 \mathrm{~m}$ on the other sets due to geology and tectonic.

\section{Conclusions}

Geostructures, textures, morphologies typically span huge ranges of scale and include structures whose direction and elongation depend on position as well as scale. However, it is frequently found that spectra $P(\underline{k})$ - an average over all the structures with a given orientation and scale (fixed wave vector $\underline{\mathrm{k}}$ ) - are not so strongly anisotropic so that the usual angle integrated spectrum $E(k)$ is nearly a (scaling) power law. In addition, the exponent of $E(k), \beta$, may be nearly the same even though the morphologies are distinct. In this case, different geomorphologies are thus associated with either differences between higher order isotropic statistics 
A)

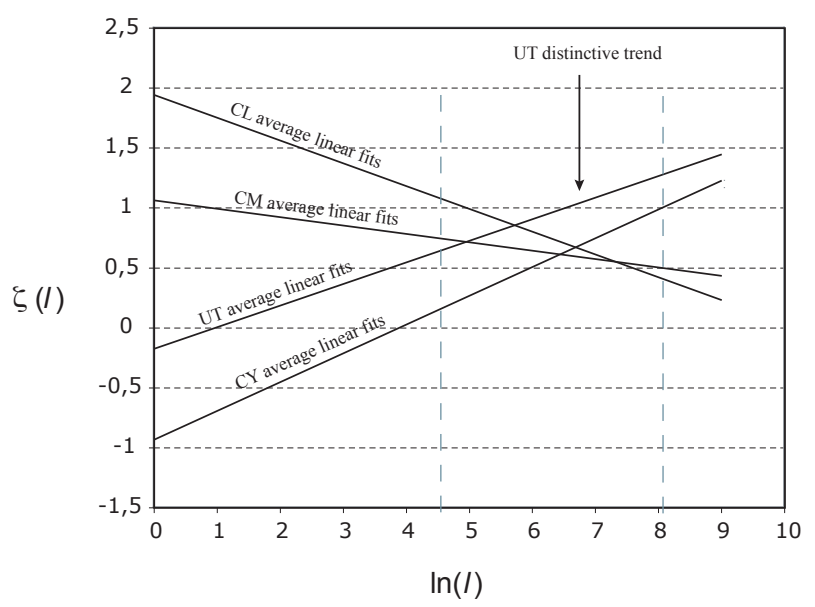

B)

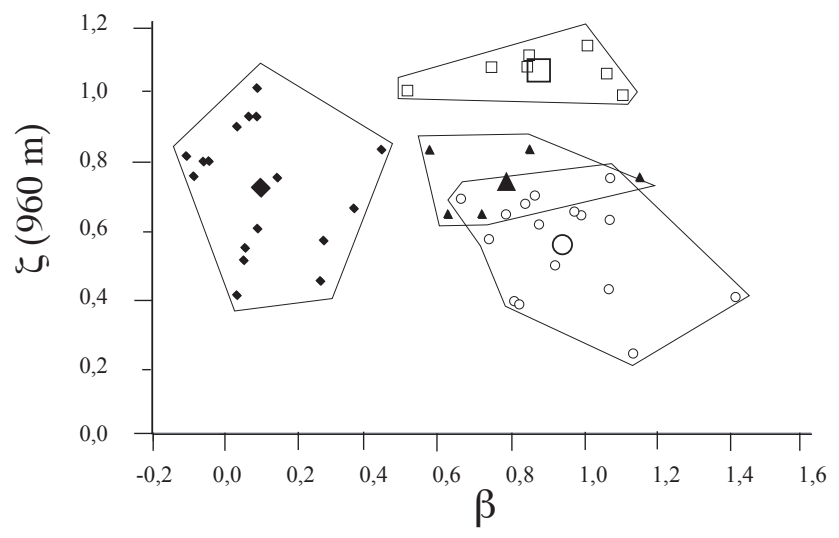

Fig. 9. (a) Plot $\zeta(l)$ versus $\ln (l)(l$ in meters) for each data group using average $\zeta_{1}$ and $\zeta_{0}$. A distinct UT region with stronger stratification is exhibited between $\ln (l)=6$ and $\ln (l)=8$. The dashed lines represent the limits of the observed range of scales; (b) Plot of $\zeta(960)$ values versus the $\beta$ values estimated from $P(\underline{k})$ for the different data ensembles. Symbols are the same as in Fig. 8.

(i.e. their multiscaling properties: the power spectrum is only a second order statistic) or different anisotropies or both. In this paper, we focus on characterizing the anisotropy by developing a new "Differential Anisotropic Scaling" technique. The method works by approximating structures in $P(\underline{k})$ by ellipses and then systematically determining how their orientations and elongations vary with scale and characterizing each of these by linear regression. Whether or not scaling holds, the method provides a valid and useful characterization of the differential anisotropy. However, if the anisotropy is not too strong, then the resulting parameters have a relatively simple interpretation in the GSI framework. The method quantifies the statistical anisotropy so that while the individual real space structures may be highly elongated (anisotropic) they nevertheless do not necessarily have an overall preferential direction. The extracted parameters are therefore independent of the approximate scaling of the isotropic statis- tics (such as the angle integrated power spectrum). With respect to existing anisotropy exponent estimation techniques, this method avoids the problems associated with nonlinear regressions in high dimensional parameter spaces.

In order to test out the method, we considered the example of erosional landforms. The type of soil and rock basement, the tectonics, the climate and the vegetation all influence the erosion mechanism, the permeability of the superficial deposits and hence the resulting river network geometries. All of these mechanisms affect satellite radiances over huge ranges of scale and hence should be reflected in remotely sensed images. In this study, we attempted to use anisotropic scaling parameters to classify TERRA ASTER satellite images of the semi-arid, vegetation free environments of the Loess Plateau, China and Desolation Canyon, Utah.

We first confirmed that the images did indeed exhibit isotropic and anisotropic scaling from $240 \mathrm{~m}$ to $7680 \mathrm{~m}$. The isotropic $\beta$ values simply express the relative importance of small and large structures. Superfine loess dentritic textures (CL images) exhibiting predominantly small source tributaries were distinguished by their relatively small $\beta$ 's. The three other image sets form a high $\beta$ group without distinction on the basis of their spectral exponent. To be more discriminating, anisotropic analyses were performed. A particularly useful parameter is the differential anisotropy tendency $\zeta_{1}$, which quantifies the rate at which structures change their elongations as functions of scale. It was found that this parameter distinguishes between images with large welloriented structures such as rivers and between those without preferential orientations such as source tributaries. Finally the elongation at the reference scale of $960 \mathrm{~m}(\zeta(960))$ is well correlated with variations in the Earth basement due to differences in lithology (loess versus sedimentary rocks), tectonics and erosion/deposition processes and provided additional classification information.

These promising results open new avenues for both understanding quantitative geomorphological classification/segmentation using satellite or spacecraft images. While further research is needed on the subject of regional variations of anisotropic exponents (i.e. spatially varying G's, non linear GSI), we also need a more systematic theory of weak scaling anisotropy. Also needed are generalizations to stronger differential anisotropy as well as to anisotropic fields that could not be well described via elliptical Fourier space structures. Analyses of remotely sensed data from other spectral regions could moreover be investigated to better constrain the properties of the studied drainage basins.

Acknowledgements. This research has been funded by the National Sciences and Engineering Research Council of Canada. We are grateful to GEOTOP centre and UQAM for scholarships to A. Beaulieu. We would also like to thank the Land Processes Distributed Active Archive Center and the NASA's Earth Observing System (EOS) Data and Information System (EOSDIS) initiative for the availability of 1999-2001 free TERRA ASTER data. GEOTOP publication no. 2007-0018. 
Edited by: A. Tarquis

Reviewed by: I. de Lima and A. A. Cârsteanu

\section{References}

Beaulieu, A. and Gaonac'h, H.: Scaling of differentially eroded surfaces in the drainage network of the Ethiopian plateau, Rem. Sens. Environ., 82, 111-122, 2002.

Chase, C. G.: Fluvial landsculpting and the fractal dimension of topography, Geomorphology, 5, 39-57, 1992.

Cheng, Q.: A new model for quantifying anisotropic scale invariance and for decomposition of mixing patterns, Mathematical Geology, 36, 345-360, 2004.

Derbyshire, E.: Geological hazards in loess terrain, with particular reference to the loess regions of China, Earth-Sci. Rev., 54, 231260, 2001

Dickinson, W. R., Lawton, T. F., and Inman, K. F.: Sandstone detrical modes, central Utah foreland region: stratigraphic record of cretaceous-paleogene tectonic evolution, J. Sedimentary Geology, 56, 276-293, 1986.

Foufoula-Georgiou, E., and Sapozhnikov, B.: Anisotropic scaling in braided rivers: an integrated theoretical framework and results from application to an experimental river, Water Resour. Res., 34, 863-867, 1998.

Franczyk, K. J., Hanley, J. H., Pitman, J. K., and Nichols, D. J.: Paleocene depositional systems in the western Roan Cliffs, Utah, Utah Geol. Ass. Pub., 19, 111-127, 1991.

Gagnon, J.-S., Lovejoy, S., and Schertzer, D.: Multifractal Earth topography, Nonlin. Processes Geophys., 13, 541-570, 2006, http://www.nonlin-processes-geophys.net/13/541/2006/.

Gaonac'h, H., Lovejoy, S., and Stix, J.: The resolution dependence of basaltic lava flows and their fractal dimensions, Geophys. Res. Lett., 19, 785-788, 1992.

Gaonac'h, H., Lovejoy, S., and Schertzer, D.: Resolution dependence of infrared imagery of active thermal features at Kilauea volcano, Int., J. Rem. Sens., 24, 2323-2344, 2003.

Harvey, D. C., Gaonac'h, H., Lovejoy, S., and Schertzer, D.: Multifractal characterization of remotely sensed volcanic features: a case study from Kilauea volcano, Hawaii, Fractals, 10, 265-274, 2002.

Horton, R. E.: Erosional development of streams and their drainage basins: hydrophysical approach to quantitative morphology, Geol. Soc. Am. Bull., 56, 275-370, 1945.

La Barbera, P. and Rosso, R.: On the fractal dimension of stream networks, Water Resour. Res., 25, 735-741, 1989.

Laferrière, A. and Gaonac'h, H.: Multifractal properties of visible reflectance fields from basaltic volcanoes, J. Geophys. Res., 104(B3), 5115-5126, 1999.

Lavallée, D., Lovejoy, S., Schertzer, D., and Ladoy, P.: Nonlinear variability of Landscape topography: Multifractal analysis and simulation, in: Fractals in Geography, edited by: De Cola, L. and Lam, N., pp. 158-192, Prentice-Hall, Englewood Cliffs, NJ, 1993.

Lewis, G. M., Lovejoy, S., Schertzer, D., and Pecknold, S.: The scale invariant generator technique for quantifying anisotropic scale invariance, Comput. Geosci., 25, 963-978, 1999.

Lilley, M., Lovejoy, S., Strawbridge, K., and Schertzer, D.: 23/9 dimensional anisotropic scaling of passive ad- mixtures using lidar data of aerosols, Phys. Rev. E, 70, doi:10.1103/PhysRevE.70.036307, 2004.

Lovejoy, S. and Schertzer, D.: Generalized scale invariance in the atmosphere and fractal models of rain, Water Resour. Res., 21, 1233-1250, 1985.

Lovejoy, S. and Schertzer, D.: Our multifractal atmosphere: a unique laboratory for nonlinear dynamics, Phys. Can., 46(4), 62 71,1990

Lovejoy, S., Schertzer, D., and Pflug, K.: Generalized scale invariance and differential rotation in cloud radiances, Physica A, 185, $121-128,1992$.

Lovejoy, S., Pecknold, S., and Schertzer, D.: Stratified multifractal magnetization and surface geomagnetic fields-I. Spectral analysis and modelling, Geophys. J. Int., 145, 112-126, 2001a.

Lovejoy, S., Schertzer, D., Tessier, Y., and Gaonac'h, H.: Multifractals and resolution-independent remote sensing algorithms: the example of ocean colour, Proc. Int. J. Rem. Sens., 22, 11911234, 2001b.

Lovejoy, S. D., Schertzer, J., and Gagnon, S.: Multifractal simulations of the Earth's surface and interior: anisotropic singularities and morphology, in GIS and Spatial Analysis, Proc. Of Inter. Assoc. Math. Geology, edited by: Cheng, Q. and Bonham-Carter, G., 37-54, 2005.

Maître, H. and Pinciroli, M.: Fractal characterization of a hydrological basin using SAR satellite images, IEEE Trans. Geosci. Rem. Sens., 37, 175-181, 1999.

Pecknold, S., Lovejoy, S., Schertzer, D., and Hooge, C.: Multifractals and resolution dependence of remotely sensed data: GSI to GIS, in: Scale in Remote Sensing and GIS, edited by: Quattrochi, D. A. and Goodchild, M. F., pp. 361-394, Boca Raton, FL, CRC Press, 1997.

Pecknold, S., Lovejoy, S., and Schertzer, D.: Stratified multifractal magnetization and surface geomagnetic fields-II. Multifractal analysis and simulations, Geophys. J. Int., 145, 127-144, 2001.

Pelletier, J. D.: Self-organisation and scaling relationships of evolving river networks, J. Geophys. Res., 104, 7395-7375, 1999.

Pflug, K., Lovejoy, S., and Schertzer, D.: Differential rotation and cloud texture: analysis using generalized scale invariance, J. Atmos. Sci., 50, 538-553, 1993.

Porter, S. C.: Chinese loess record of monsoon climate during the last glacial-interglacial cycle, Earth-Sci. Rev., 54, 115-128, 2001.

Rodriguez-Iturbe, I. and Rinaldo, A.: Fractal river basins: Chance and self-organisation, 564 pp. Cambridge, UK, Cambridge Univ. Press, 2001.

Schertzer, D. and Lovejoy, S.: Generalised scale invariance in turbulent phenomena, Phys. Chem. Hydrodyn. J., 6, 623-635, 1985.

Veneziano, D. and Iacobellis, V.: Self-similarity and multifractality of topographic surfaces at basin and subbasin scales, J. Geophys. Res., 104, 12 797-12 812, 1999.

Wang, Q. and Takahashi, H.: Regional hydrological effects of grassland degradation in the Loess Plateau of China, Hydrol. Processes, 12, 2279-2288, 1998.

Weissel, J. K. and Pratson, L. F.: The length-scaling properties of topography, J. Geophys. Res., 99, 13 997-14 012, 1994.

Weissel, J. K., Malinverno, A., Harding, D. J., and Karner, G. D.: Erosional development of the Ethiopian Plateau of northeast Africa from a fractal analysis of topography, in: Fractals in Petroleum Geology and Earth Processes, edited by: Barton, C. 
A. Beaulieu et al.: Anisotropic scaling of remotely sensed drainage basins

C. and La Pointe, P. R., pp. 127-142, Plenum Press, New York, 1995.
Zunyi, Y., Yuqi, C., and Hongzhen, W.: The Geology of China, Oxford Monographs on Geology and Geophysics no. 3, 303 pp., Clarendon Press, Oxford, 1986. 